\title{
Higher-Order Demand-Driven Program Analysis
}

\author{
Zachary Palmer ${ }^{1}$ and Scott F. Smith ${ }^{2}$
}

1 Swarthmore College

Swarthmore, PA, USA

zachary.palmer@swarthmore.edu

2 The Johns Hopkins University

Baltimore, MD, USA

scott@cs.jhu.edu

\begin{abstract}
We explore a novel approach to higher-order program analysis that brings ideas of on-demand lookup from first-order CFL-reachability program analyses to higher-order programs. The analysis needs to produce only a control-flow graph; it can derive all other information including values of variables directly from the graph. Several challenges had to be overcome, including how to build the control-flow graph on-the-fly and how to deal with non-local variables in functions. The resulting analysis is flow- and context-sensitive with a provable polynomial-time bound. The analysis is formalized and proved correct and terminating, and an initial implementation is described.
\end{abstract}

1998 ACM Subject Classification F.3.2. Semantics of Programming Languages

Keywords and phrases functional programming, program analysis, polynomial-time, demanddriven, flow-sensitive, context-sensitive

Digital Object Identifier 10.4230/LIPIcs.ECOOP.2016.19

Supplementary Material ECOOP Artifact Evaluation approved artifact available at http://dx.doi.org/10.4230/DARTS.2.1.9

\section{Introduction}

Flow analysis for imperative first-order programs is a well-known and straightforward process. Before the analysis starts, it is known which functions are being invoked at each call site in the source program. This is possible because, in absence of higher-order functions, potential control flow is determined immediately from the structure of the program. So, for first-order programs, it is possible to directly build a fixed control flow graph (CFG) where each edge in the CFG points to a potential next program point. The program analysis then can monotonically accumulate information about what values program variables could take on at each program point, propagating information along this fixed CFG.

\section{Forward higher-order program analysis}

In languages with higher-order functions, program analysis is much more challenging: it is no longer obvious which functions may be invoked at each call site. The program's data flow determines which functions appear at the call site, in turn influencing the program's control flow. Accurate analyses of programs with higher-order functions must therefore compute data- and control-flow information simultaneously $[12,16]$.

Higher-order program analyses are generally based on abstract interpretations [2]; such analyses define a finite-state abstraction of the operational semantics transition relation to 
soundly approximate the program's runtime behavior. The resulting analysis has the same general structure as the operational semantics it was based on: program points, environments, stacks, stores, and addresses are replaced with abstract counterparts which have finite cardinality, "hobbling" the full operational semantics of the language to guarantee termination of the analysis [13]. A sound analysis will visit the (finitely many) abstract counterparts of all reachable concrete program states, producing a finite automaton representing all potential program runs.

We use the term "forward analyses" to refer to standard higher-order program analyses, to emphasize how they propagate data forward through the program in the same manner as an operational semantics does. All abstract interpretation based higher-order program analyses, including $[12,16,22,13,27,8,15,3]$, are forward analyses.

Since each node is an abstract state and there can be a great many combinations of data for environment or store, even the finitized abstract state space can be very large. For this reason, any practical analysis must compress the total number of states. Typically, the program counter is preserved. One standard compression, store widening, replaces the store in each each abstract program state with a single global store; this global store is then the union of all the individual stores.

Store widening is effective in compressing the abstract state set but tends to give too little precision. For example, function polymorphism which is present before store widening may be lost as the parameters to the calls of a given function in each program state are unioned together. Store widening also generally loses any so-called flow sensitivity, where a given (often stateful) variable can take on different values at different points.

So, other methods are employed to recover expressiveness without a full store in each abstract state. One method is polyvariance, such as in $k \mathrm{CFA}$ [22] for $k>0$, where each function parameter gets unique addresses relative to the $k$ most recent stack frames. Another method is call-return alignment: the analysis uses a stack internally to return only to the call site of the particular call being analyzed [26]. Call-return alignment also gives some, but not all, of the power of polyvariance. One alternative to store widening is abstract garbage collection [14], which also limits the number of abstract states: the store is not widened, but an analysis-time analogue of run-time garbage collection removes inaccessible bindings from the store. Abstract GC preserves flow-sensitivity, but this technique is still susceptible to an explosion in analysis states for programs with nontrivial amounts of persistent data as each configuration of this data represents another abstract program state to consider.

\section{A demand-driven approach to higher-order programs}

In this paper, we explore a fundamentally different approach to higher-order program analysis which does not proceed by finitizing an operational semantics. Rather than pushing data forward as in an operational semantics, the analysis looks up data on demand. This lookup walks backward along the control flow from the point at which a variable's value is needed to the point at which the variable was most recently defined. Actual values never need to be pushed forward so, in some sense, lookup is lazier than a standard lazy interpreter.

CFL-reachability analyses [6] have shown how demand-driven variable lookup is possible in first-order program analyses. In comparison to forward analyses, the demand-driven approach has potential for better performance: the aforementioned optimizations are unnecessary as no store is propagated forward through the analysis. CFL-reachability is a first-order analysis, where the CFG is already known and where there are no non-local variables in functions. Demand-driven lookup is applied to higher-order languages in [18], but in the a context of a flow-insensitive type-based analysis with other restrictions. To 
generally apply this technique to higher-order programs, we must have (1) a technique for constructing the CFG in tandem with the data flow analysis and (2) a strategy for handling non-local variables.

In this paper, we solve these two problems to produce a higher-order Demand-Driven Program Analysis (DDPA). The CFG is constructed incrementally just as in a forward higher-order program analysis. Unlike a forward analysis, we only need to construct the CFG; there is no abstracted environment/store/etc as all of the variable lookup happens in reverse by following CFG edges backwards. To deal with non-local variables in functions, we develop a novel reverse lookup process that is related to how access links are used in a compiler to look up non-local variables: we find the definition of the function itself and look up the variable from there. For added precision, we incorporate call-return alignment from CFL-reachability $[21,20]$ and pushdown higher-order flow analyses $[26,8]$.

\section{Contrast with forward analyses}

During the course of DDPA, each variable lookup occurs relative to a particular point in the (partial) control flow graph where the variable is used; this makes flow-sensitivity a natural component of DDPA. In forward higher-order analyses, such flow-sensitivity is expensive since it prohibits common performance-enhancing techniques such as store widening. Abstract garbage collection, an aforementioned alternative to store widening, prunes the stores significantly but produces many states for persistent data. Additionally, there is no need for explicit polyvariance in DDPA: the combination of call-return alignment and non-local lookup achieves the full effect of polyvariance without an explicit polyvariance model a la $k$ CFA. DDPA appears to compare favorably to forward analyses: it has the simultaneous benefits of store widening (we have no per-node store), abstract garbage collection (demanddriven lookup prevents the generation of garbage), call-return alignment (the one feature we directly adopt) and polyvariance (which is subsumed by our call-return alignment given our novel non-local variable lookup mechanism). Forward analyses cannot combine all these features because abstract GC is ineffective in the presence of store widening [8].

In theory, DDPA has minimal requirements on run-time data structures: the only required data structure is the CFG, which is minuscule compared to the formal treatment of forward analysis. In practice, an efficient DDPA implementation requires significant caching structures which are similar to fragments of an abstract store in a forward analysis. We do not assert DDPA to be a strictly better form of program analysis, only a substantially different one with different trade-offs and worthy of study.

In terms of technical overhead, DDPA has the advantage of naturally scaling up to a flow-, path-, and context-sensitive analysis while preserving a relatively compact formal specification. On the other hand, reverse lookup is a different process than the original (forward) operational semantics and requires a major re-thinking about program flow; a forward analysis can to some degree be "read off the operational semantics" [25, 13].

We have completed a proof-of-concept implementation of DDPA, described in Section 6, and include it as an artifact archived with the paper. The implementation builds a pushdown automaton (PDA) and variable lookup questions can be cast as reachability questions in the PDA. This implementation has not been optimized but it confirms that the analysis has the expected behavior on examples. 


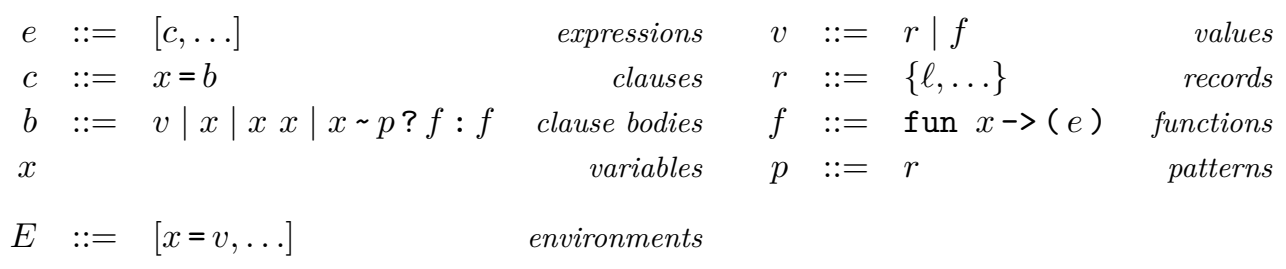

Figure 1 Expression Grammar.

\section{Paper outline}

Section 2 presents an example-driven description of the main features of the analysis. Section 3 gives the full details, and Section 4 establishes its soundness relative to an operational semantics. Section 5 defines extensions for full records, path-sensitivity, and state. Section 6 describes the implementation. Section 7 covers related work. We conclude in Section 8 .

\section{Overview of the Analysis}

This section informally presents DDPA by example.

\subsection{A Simple Language}

For a given program, our objective is to establish the possible execution paths that the program will take. We encode this information in the form of a CFG, more precisely as a happens-before relation $c \ll c^{\prime}$ : program point $c$ related to $c^{\prime}$ means there may be control flow from $c$ to $c^{\prime}$.

We use a simple functional language defined in Figure 1. To make it easier to keep track of program points and operation sequencing, we restrict our language syntax to a shallow A-normal form (ANF) [5]. We further restrict all variables to be unique; this ensures that clauses $c$ themselves denote program points. (Notation: lists are written $\left[g_{1}, \ldots, g_{n}\right]$, and $\|$ denotes list concatenation. We may overload as $g_{1} \|\left[g_{2}, g_{3}\right]=\left[g_{1}, g_{2}, g_{3}\right]$ when it is unambiguous.)

The operational semantics of this (eager) language are straightforward and are given in Section 4.1. Note that we have a degenerate form of record with label components only; this restriction is made to simplify the formalism and we relax this restriction in the implementation. Case analysis is written $x \sim p$ ? $f_{1}: f_{2}$; we execute $f_{1}$ with $x$ as argument if the value of $x$ matches the pattern $p$, and $f_{2}$ with $x$ as argument otherwise.

\subsection{The Basic Analysis}

Consider the program in Figure 2. Note that $\mathrm{f}$ is just a fancy identity function used to help illustrate the analysis, and that clause no is a "no-op": it is present only to help clarify the diagrams.

The analysis begins by constraining the top-level clauses to happen in order: $f, x 1, z 1$, $\mathrm{x} 2$, and then $\mathrm{z} 2$. For technical reasons, we also add distinguished START and END nodes.

Figure 3 shows the completed analysis; up to now we have only described the bottom row. Focusing only on that row for now, the solid arrows $(\longrightarrow)$ represent nodes ordered by $\ll$ in the source program, and indicate what we know about control flow at the beginning of the analysis: only that control can move from each clause to the next. As shorthand, we 


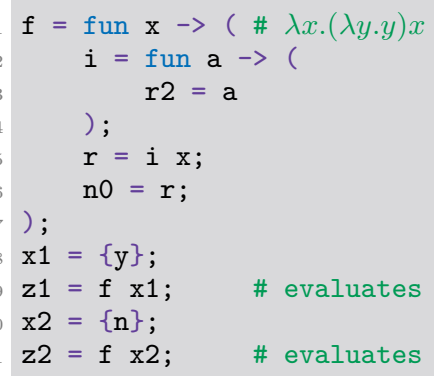

Figure 2 Invocation Example: ANF.

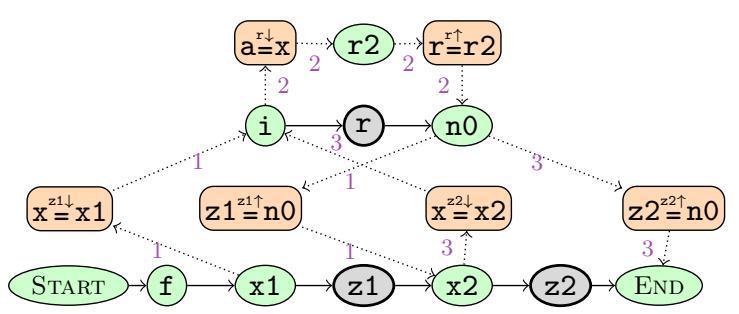

Figure 3 Invocation Example: Analysis.

identify each clause by the unique variable it assigns; thus, the green node labeled $\mathrm{x} 1$ refers to line 8 in Figure 2. Green nodes are immediate clauses and gray nodes are call sites.

\subsubsection{Adding control flows for function calls}

To elaborate the call graph, we must connect each call site to any function body invoked at that call site. Proceeding forward on the control flow from the program start, the first call site (gray node) is node $\mathbf{z} 1$. The dotted edges $(\cdots \cdots)$ annotated with the number "1" represent control passing from call site $z 1$ to the start of the body of $f$ (the $i, r, n 0$ clauses), and from the end of the function body back to $z 1$. Note that the wires in fact run "around" the call site in the figure, going out the predecessor of $z 1$ and back to the successor. This reflects how a function inlining would work. (Aside: both $\longrightarrow$ and $\cdots \rightarrow$ edges define $\ll$ relationships between program points; the two edge sorts are used for illustration only.)

Additionally, the call adds two new intermediate nodes: $\mathrm{x}=\mathrm{x} 1$ represents copying the argument $\mathrm{x} 1$ at the call site into the function $\mathrm{f}$ 's parameter, while $\mathrm{z} 1=\mathrm{n} 0$ represents copying the result of the function into the variable defined at the call site. These nodes are marked with annotations to indicate their call site and purpose; $z 1 \downarrow$, for instance, indicates a parameter passed in from the $\mathbf{z} 1$ call site. All of the information in these new nodes can in fact be inferred from context since all values are unique given a (call site, function) pair. In other words, the happens-before relation is isomorphic to the call graph.

Continuing with the analysis: now that the body of $f$ has been wired in, the call site $r$ can execute, adding two orange wiring nodes and the corresponding " 2 " flows. The " 3 " flows are similarly added when we elaborate $z 2$.

\subsubsection{Variable lookup as reverse walk}

In the description above, we glossed over how variable lookup occurs: for each call site, we needed to look up the particular functions to invoke and this can be nontrivial in a higherorder language. The clause $\mathbf{z} 1$ invokes $f$, and all potential definitions of $f$ need to be wired to the call site. This particular case is simple since $f$ obviously has only one value.

In general, variable lookup is contextual: lookup starts at the clause where the variable is being used and proceeds backwards in time with respect to the control flow to find the most recent value. This approach to lookup is related to the demand-driven optimizations of first-order CFL-reachability analyses [6].

For a less trivial example, let us look up $\mathrm{x}$ from the perspective of $r$ 's defining clause in line 5. Walking back though the graph from $r$, there are two definitions of $\mathrm{x}$ reached: 
$\mathrm{x}=\mathrm{x} 1$ and $\mathrm{x}=\mathrm{x} 2$. Since $\mathrm{x} 1 / \mathrm{x} 2$ are not concrete values, lookup proceeds by looking up those variables, respectively. Ultimately, $\mathrm{x}$ has final value set $\{\{\mathrm{y}\},\{\mathrm{n}\}\}$, the set of arguments the function was invoked on.

Since variable lookup always starts at the node representing the redex where the variable is used, we obtain a flow-sensitive analysis by default. Note that the specification of lookup traverses all the way back to the original definition every time; for example, in looking up $\mathrm{x}$ we needed to look up $\mathrm{x}=\mathrm{x} 1$ and we then needed to continue back to $\mathrm{x} 1$ 's original definition. An efficient implementation should cache, but that is orthogonal to the specification.

\subsection{Constraining lookup to reasonable call stacks}

The above description of a simple lookup leaves out an important refinement in DDPA: it is possible to rule out variable lookup search paths on which calls and returns do not align, because such a path corresponds to no program execution. To show the incompleteness of lookup as described thus far, consider how lookup would find values for $\mathrm{z} 2$ from the perspective of the end of the program. To find $z 2$, we proceed back on the control flow to $\mathrm{z} 2=\mathrm{n} 0$ (recall how the call site itself is dead code after all calls have been wired in), at which point the search is now for the value of $n 0$; continuing back we reach $n 0=r$ and proceed by looking up $\mathrm{r}$ from node $\mathrm{n}$, and so on until we are looking up $\mathrm{x}$ from node $\mathrm{i}$. Here, there are two paths, to either $\mathrm{x}=\mathrm{x} 1$ or $\mathrm{x}=\mathrm{x} 2$. So, we take both paths and union the result, obtaining $\{\{\mathrm{y}\},\{\mathrm{n}\}\}$. This is clearly a loss of precision: $\{\mathrm{y}\}$ cannot appear as the argument of call site z2 at runtime. But, looking at the overall path we took above in this spurious lookup, we walked back into the function via the $z 2$ call site and came out of the front of the function to the $z 1$ call site. This is a non-sensical program execution path since the call and return site are not aligned.

The annotations $z 1 \downarrow / z 1 \uparrow$ on the wiring nodes indicate a call into and return from call site $z 1$, respectively, and are used to filter out these spurious paths. On any lookup path the wiring annotations must pair correctly; that is, one may only visit a $z 1 \downarrow$ node if a corresponding $z 1 \uparrow$ node was visited. We track these pairings using an abstract call stack to verify they obey a reasonable call-return discipline. The idea of call-return alignment is taken from CFL-reachability [21, 20] and higher-order pushdown analyses [26, 8].

Note that the above analysis exhibits polyvariant behavior: different invocations of the same function are not analyzed uniformly. Polyvariance is commonly achieved by copying, $k$ CFA being a canonical example [16]. In DDPA, polyvariance can be achieved solely by call-return alignment, unlike [26, 8] - stack alignment in these analyses only aligns variables local to the function body, whereas DDPA also aligns non-locals; the subtleties of non-local lookup are covered next.

\subsection{Looking up Non-Local Variables}

So far, our examples of lookup have been restricted to local variables. However, the above lookup process does not accurately reflect how non-local variables are bound: if non-local variables were handled in the same way, lookup would give non-locals a dynamically scoped semantics. To see why, consider the code in Figure 4 and its graph in Figure 5. If we look up the value of $z$ from the end of the program, we find ourselves asking for the values of $v$ at the wiring of $j=e$. Proceeding as described above, $j$ does not match $v$ and we would attempt to find a value for $\mathrm{v}$ at the e clause. This leads us to the clause $\mathrm{v}=\mathrm{b}$, which in turn gives us just the value $\{\mathrm{b}\}$. This would be unsound as it is not consistent with run-time behavior. 


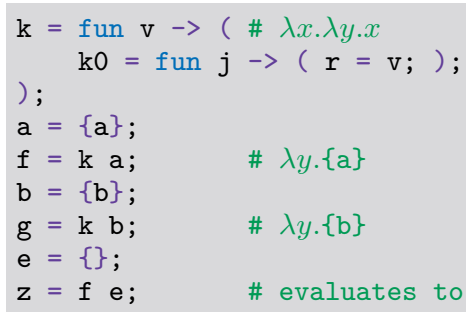

Figure 4 Non-Local Variable: ANF.

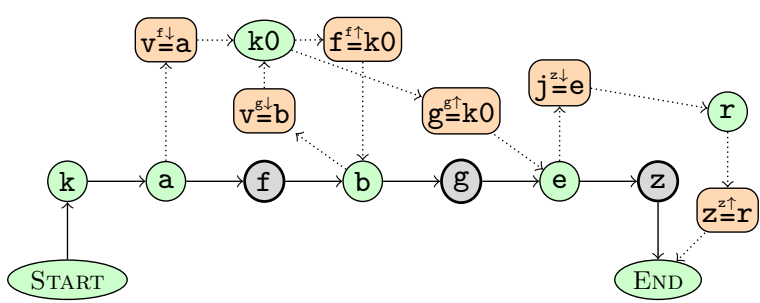

Figure 5 Non-Local Variable: Analysis.

To give lexical scoping semantics, the lookup function must be modified. Lexical scoping means we want the values of non-local variables at the point which the function containing them was itself declared, so we proceed by first locating the function definition and then resuming our search for the value of the variable. This is similar to the access link method of non-local lookup in a compiler implementation. In the example above, the pivotal decision is made when finding values of $\mathrm{v}$ when we have searched through the whole body of the function in lines 2-4 and arrived at the argument wiring $j=e$. From the annotation $z \downarrow$ on this node, we can deduce that we are leaving the current function which was called from site $\mathbf{z}$. Since we have not yet found $\mathrm{v}$, it must be a non-local. So, we then delay our search for $\mathrm{v}$, examine the call site $\mathbf{z}$ (appearing in the wiring annotation), and look up the function invoked at that point; here, this means to search for $f$ starting from e. This search leads us through $f=k 0$ to $k 0$, the point at which the function was originally defined, and from here the search for $\mathrm{v}$ can soundly resume. Using this approach, the lookup of $\mathrm{z}$ from the end of the program yields $\{a\}$.

The general case of non-local lookup chains on the above idea, since the defining function could itself be non-locally defined. The chain is up the lexical scoping structure, so its length is bound by program size.

\subsection{Looking up Higher-Order Functions}

The analysis described thus far also does not deal well with the case of multiple functions showing up at a call site - it does not take in account which functions are actually available. Consider the code example in Figure 6.

We would like a search for $r b$ from the END to conclude $\{\{b\}\}$, the run-time result. In the process of trying to answer that question, the analysis eventually gets to node no, querying for the variable $\mathrm{hr}$. At that point, there are two paths by which to proceed: one to $\mathrm{hr}=\mathrm{a}$ and another to $h r=b$. If the analysis took both paths, the result would be $\{\{a\},\{b\}\}$, but $\{$ a $\}$ will never arise at runtime.

We address this case as follows. Before entering a function call (in reverse), the analysis first runs a subordinate lookup for all functions that could show up at the call site under consideration. The main lookup can then use this result to rule out any function definition that could have not reached the call site in the current context. In the given example, at the node $\mathrm{n} 0$, before entering node $\mathrm{hr}=\mathrm{a}$ or $\mathrm{hr}=\mathrm{b}$, the analysis performs a subordinate lookup for $\mathrm{f}$ from $\mathrm{hr}$, under the current calling context of $\mathrm{rb}$. In order to align with the $\mathrm{rb}=\mathrm{n} 0$ entrance node, the only possible answer is $\mathrm{fb}$, ruling out the choice of $\mathrm{hr}=\mathrm{a}$ and leading to a final answer of only $\{b\}$. 

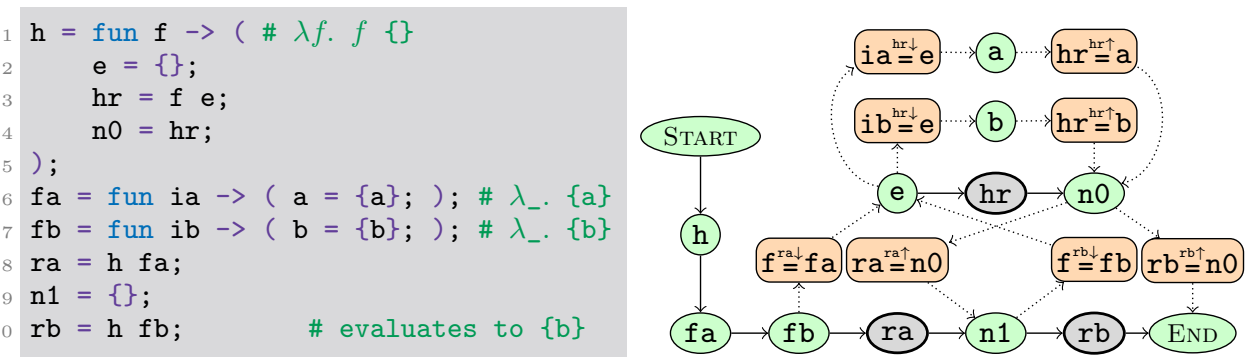

Figure 6 Higher-Order Functions: ANF.

Figure 7 Higher-Order Functions: Analysis.

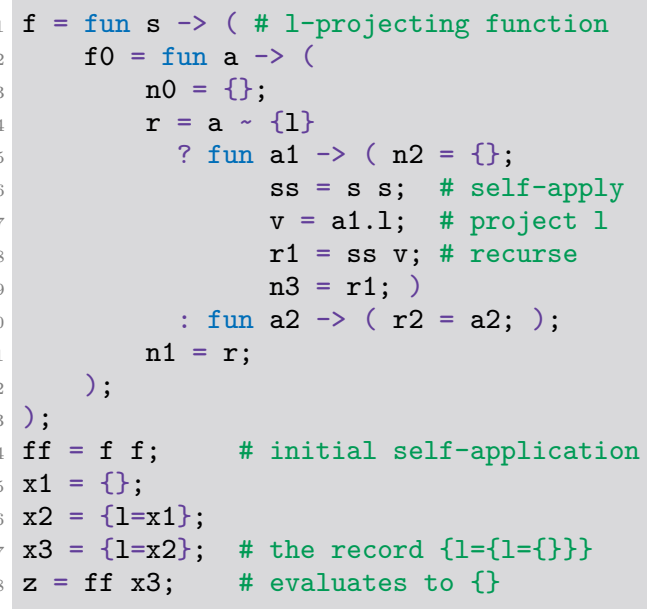

Figure 8 Recursion: ANF.

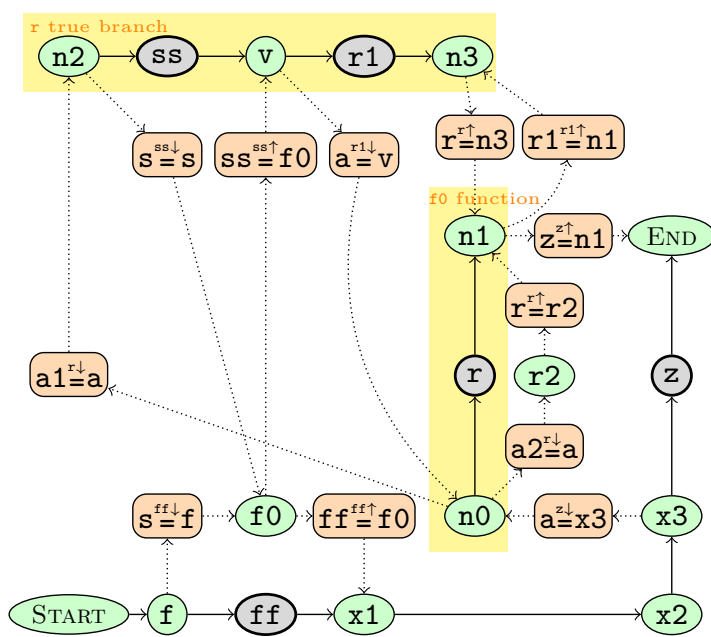

Figure 9 Recursion: Analysis.

\subsection{Recursion and Decidability}

The analysis described above naturally analyzes recursive programs: consider the program in Figure 8 which we code in an extension including proper records. This program defines a function $f$ which is recursive (by self-passing) and which deeply projects any number of 1 fields from a record.

The DDPA result appears in Figure 9. Lookup generally proceeds via the same process as for the previous examples. The only complication with recursion is that some control flow paths could be cyclic. For instance, the path $\mathrm{n} 0 \rightsquigarrow \mathrm{n} 2 \rightsquigarrow \mathrm{v} \rightsquigarrow \mathrm{n} 0$ is a cycle representing an arbitrary number of recursive unwrappings, each of which pushes $r \downarrow$ and $r 1 \downarrow$ onto the stack. The specification of the analysis only requires variable lookup paths to be balanced in calls and returns, so there are arbitrarily many possible paths. It is clear in this example that the number of times around the cycle can be bounded without changing the result; as we will discuss later, it is not known whether lookup over an arbitrary-size call stack is computable. We address this issue in Section 3 by defining a stack finitization $k$ DDPA which retains the $k$ most recent contexts in the spirit of $k \mathrm{CFA}$ [22].

DDPA is flow-sensitive and context-sensitive. However, it is not path-sensitive; that is, a variable's values are not considered in terms of how branching decisions at conditionals led to a particular program point. Consider for example the values of $\mathbf{z}$ which may appear 


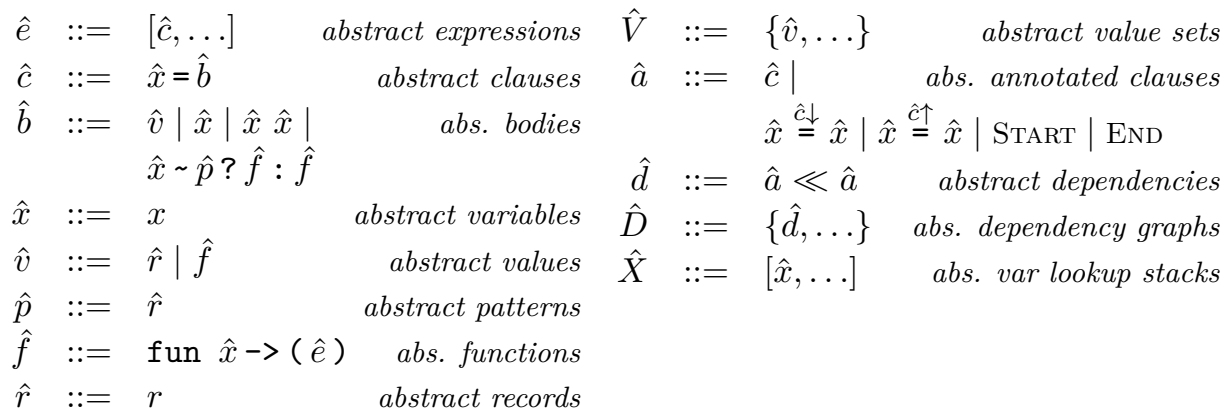

Figure 10 Analysis Grammar.

at the end of the program of Figure 8: the path $\mathrm{x} 3 \rightsquigarrow \mathrm{n} 0 \rightsquigarrow \mathrm{r} 2 \rightsquigarrow \mathrm{n} 1 \rightsquigarrow$ END in Figure 9 is valid, but this would imply that the value $\{l=\{l=\{\}\}\}$ is possible for $z$, but it will not arise at runtime. Path sensitivity can close this gap, and we sketch such an extension in Section 5.2. Note this example also uses "real" records and not just the label sets of our grammar of Figure 1; we sketch an extension to full records in Section 5.1.

\section{The Analysis Formally}

In this section we formalize the analysis algorithm. The operational semantics is standard and so we postpone it and the soundness proof to Section 4.

The grammar constructs needed for the analysis appear in Figure 10. The items on the left are just the hatted versions of the corresponding program syntax. As mentioned above, we require variables to be bound uniquely so that each program point is defined by exactly one variable. Furthermore, we assume the expressions we analyze to be closed: a variable is not used until after the clause in which it is bound.

Edges in a dependency graph $\hat{D}$ are dependencies $\hat{d}$, are written $\hat{a} \ll \hat{a}^{\prime}$ and mean clause $\hat{a}$ happens right before clause $\hat{a}^{\prime}$. New clause annotations $\hat{c} \downarrow / \hat{c} \uparrow$ are used to mark the entry and exit points for functions/cases.

- Definition 3.1. We use the following notational sugar for graph dependencies:

- We write $\hat{a}_{1} \ll \hat{a}_{2} \ll \ldots \ll \hat{a}_{n}$ to mean $\left\{\hat{a}_{1} \ll \hat{a}_{2}, \ldots, \hat{a}_{n-1} \ll \hat{a}_{n}\right\}$.

- We write $\hat{a}^{\prime} \ll\left\{\hat{a}_{1}, \ldots, \hat{a}_{n}\right\}$ (resp. $\left.\left\{\hat{a}_{1}, \ldots, \hat{a}_{n}\right\} \ll \hat{a}^{\prime}\right)$ to denote $\left\{\hat{a}^{\prime} \ll \hat{a}_{1}, \ldots, \hat{a}^{\prime} \ll \hat{a}_{n}\right\}$ (resp. $\left\{\hat{a}_{1} \ll \hat{a}^{\prime}, \ldots, \hat{a}_{n} \ll \hat{a}^{\prime}\right\}$ ).

- We write $\hat{a} \ll \hat{a}^{\prime}$ to mean $\hat{a} \ll \hat{a}^{\prime} \in \hat{D}$ for some graph $\hat{D}$ understood from context.

- We define abbreviations $\operatorname{Preds}(\hat{a})=\left\{\hat{a}^{\prime} \mid \hat{a}^{\prime} \ll \hat{a}\right\}$ and $\operatorname{Succs}(\hat{a})=\left\{\hat{a}^{\prime} \mid \hat{a} \ll \hat{a}^{\prime}\right\}$.

- Definition 3.2. Initial embedding $\operatorname{EmbeD}\left(\left[c_{1}, \ldots, c_{n}\right]\right)$ is the graph $\hat{D}$ given by Start $\ll$ $\hat{c}_{1} \ll \ldots \ll \hat{c}_{n} \ll$ END, where each $\hat{c}_{i}=c_{i}$.

This initial graph is just the linear sequence of clauses in the "main program".

\subsection{Lookup}

As was described in Section 2, the analysis will look back along « edges in the graph $\hat{D}$ to search for definitions of variables it needs. We now define this lookup function. 


\subsubsection{Context Stacks}

The definition of lookup proceeds with respect to a current context stack $\hat{C}$. The context stack is used to align calls and returns to rule out cases of looking up a variable based on a non-sensical call stack, and was described in Section 2.3.

The proof of decidability relies upon bounding the depth of the call stack. We first define a general call stack model for DDPA, and in Section 3.3 below we instantiate the general model with a fixed $k$-depth call stack version notated $k$ DDPA; this is a simple bounding strategy and our model can in principle work with other strategies.

- Definition 3.3. A context stack model $\Sigma=\langle\hat{\boldsymbol{C}}, \epsilon$, Push, Pop, IsTop $\rangle$ obeys the following:

1. $\hat{C}$ is a set. We use $\hat{C}$ to range over elements of $\hat{C}$ and refer to such $\hat{C}$ as context stacks.

2. $\epsilon \in \hat{\boldsymbol{C}}$.

3. $\operatorname{Push}(\hat{c}, \hat{C})$ and $\operatorname{Pop}(\hat{C})$ are total functions returning stacks.

4. $\operatorname{IsTop}(\hat{c}, \operatorname{Push}(\hat{c}, \hat{C}))$, IsTop $(\hat{c}, \epsilon)$, and $\operatorname{Pop}(\epsilon)=\epsilon$ hold.

5. If $\operatorname{IsTop}(\hat{c}, \hat{C})$ then $\operatorname{IsTop}\left(\hat{c}, \operatorname{Pop}\left(\operatorname{Push}\left(\hat{c}^{\prime}, \hat{C}\right)\right)\right)$.

The context stack represents the calls of which we are certain; thus, the empty stack represents "no knowledge" (and not "no stack frames"). Thus, popping from the empty stack yields the empty stack and any clause is considered to be on top of an empty stack.

\subsubsection{Lookup stacks}

Lookup also proceeds with respect to a non-locals lookup stack $\hat{X}$ which is used to remember non-local variable(s) we are in the process of looking up while searching for the lexically enclosing context where they were defined. In the general case it is a stack since the function itself could prove to be a non-local, etc. This stack can be unbounded unlike the context stack above. Section 2.4 gave motivation and examples for non-local variable lookup.

\subsubsection{Defining the lookup function}

Lookup finds the value of a variable starting from a given graph node. Given a dependency graph $\hat{D}$, we write $\hat{D}\left(\hat{x}, \hat{a}_{0}, \hat{X}, \hat{C}\right)$ to denote the lookup of a definition of the variable $\hat{x}$ in $\hat{D}$ relative to graph node $\hat{a}_{0}$, access stack $\hat{X}$ and context stack $\hat{C}$. We let $\hat{D}\left(\hat{x}, \hat{a}_{0}\right)$, the top-level lookup of $\hat{x}$ from graph node $\hat{a}_{0}$, abbreviate $\hat{D}\left(\hat{x}, \hat{a}_{0},[], \epsilon\right)$. Note that we have oriented the definition so looking from graph node $\hat{a}_{0}$ means we are not looking for the value in that node itself, but in (all) predecessors of it.

- Definition 3.4. Given dependency graph $\hat{D}, \hat{D}\left(\hat{x}, \hat{a}_{0}, \hat{X}, \hat{C}\right)$ is the function returning the least set of values $\hat{V}$ satisfying the following conditions:

1. If $\hat{a}_{1}=(\hat{x}=\hat{v}), \hat{a}_{1} \ll \hat{a}_{0}$, and $\hat{X}=[]$, then $\hat{v} \in \hat{V}$.

2. If $\hat{a}_{1}=(\hat{x}=\hat{f}), \hat{a}_{1} \ll \hat{a}_{0}$ and $\hat{X}=\left[\hat{x}_{1}, \ldots, \hat{x}_{n}\right]$ for $n>0$, then $\hat{D}\left(\hat{x}_{1}, \hat{a}_{1},\left[\hat{x}_{2}, \ldots, \hat{x}_{n}\right], \hat{C}\right) \subseteq \hat{V}$.

3. If $\hat{a}_{1}=\left(\hat{x}=\hat{x}^{\prime}\right)$ and $\hat{a}_{1} \ll \hat{a}_{0}$, then $\hat{D}\left(\hat{x}^{\prime}, \hat{a}_{1}, \hat{X}, \hat{C}\right) \subseteq \hat{V}$.

4a. If $\hat{a}_{1}=\left(\hat{x} \stackrel{\hat{c} \downarrow}{=} \hat{x}^{\prime}\right), \hat{a}_{1} \ll \hat{a}_{0}, \hat{c}$ is an application clause, and $\operatorname{IsTop}(\hat{c}, \hat{C})$, then $\hat{D}\left(\hat{x}^{\prime}, \hat{a}_{1}, \hat{X}, \operatorname{Pop}(\hat{C})\right) \subseteq \hat{V}$.

4b. If $\hat{a}_{1}=\left(\hat{x} \stackrel{\hat{c} \downarrow}{=} \hat{x}^{\prime}\right), \hat{a}_{1} \ll \hat{a}_{0}$ and $\hat{c}$ is a conditional clause, then $\hat{D}\left(\hat{x}^{\prime}, \hat{a}_{1}, \hat{X}, \hat{C}\right) \subseteq \hat{V}$.

5a. If $\hat{a}_{1}=\left(\hat{x} \stackrel{\hat{c} \uparrow}{=} \hat{x}^{\prime}\right), \hat{a}_{1} \ll \hat{a}_{0}$ and $\hat{c}=\left(\hat{x}_{r}=\hat{x}_{f} \hat{x}_{v}\right)$, then $\hat{D}\left(\hat{x}^{\prime}, \hat{a}_{1}, \hat{X}, \operatorname{PusH}(\hat{c}, \hat{C})\right) \subseteq \hat{V}$, provided fun $\hat{x}^{\prime \prime} \rightarrow(\hat{e}) \in \hat{D}\left(\hat{x}_{f}, \hat{c},[], \hat{C}\right)$ and $\hat{x}^{\prime}=\operatorname{RV}(\hat{e})$.

5b. If $\hat{a}_{1}=\left(\hat{x} \stackrel{\hat{c} \uparrow}{=} \hat{x}^{\prime}\right), \hat{a}_{1} \ll \hat{a}_{0}$ and $\hat{c}$ is a conditional clause, then $\hat{D}\left(\hat{x}^{\prime}, \hat{a}_{1}, \hat{X}, \hat{C}\right) \subseteq \hat{V}$.

6. If $\hat{a}_{1}=\left(\hat{x}^{\prime \prime}=b\right), \hat{a}_{1} \ll \hat{a}_{0}$, and $\hat{x}^{\prime \prime} \neq \hat{x}$, then $\hat{D}\left(\hat{x}, \hat{a}_{1}, \hat{X}, \hat{C}\right) \subseteq \hat{V}$. 
7a. If $\hat{a}_{1}=\left(\hat{x}^{\prime \prime} \stackrel{\hat{c} \downarrow}{=} \hat{x}^{\prime}\right), \hat{a}_{1} \ll \hat{a}_{0}, \hat{x}^{\prime \prime} \neq \hat{x}, \hat{c}$ is an application clause, and $\operatorname{IsTop}(\hat{c}, \hat{C})$, then $\hat{D}\left(\hat{x}_{f}, \hat{a}_{1}, \hat{x} \| \hat{X}, \operatorname{Pop}(\hat{C})\right) \subseteq \hat{V}$.

7b. If $\hat{a}_{1}=\left(\hat{x}^{\prime \prime} \stackrel{\hat{c} \downarrow}{=} \hat{x}^{\prime}\right), \hat{a}_{1} \ll \hat{a}_{0}, \hat{x}^{\prime \prime} \neq \hat{x}$ and $\hat{c}$ is a conditional clause, then $\hat{D}\left(\hat{x}, \hat{a}_{1}, \hat{X}, \hat{C}\right) \subseteq \hat{V}$. (where in the above $\operatorname{RV}(\hat{e})=\hat{x}$ if $\hat{e}=[\hat{c}, \ldots, \hat{x}=\hat{b}]$, i.e. $\hat{x}$ is the return variable of $\hat{e}$.)

Note this is a well-formed inductive definition by inspection. Each of the clauses above represents a different case in the reverse search for a variable; we now give clause-by-clause intuitions. (1) We finally arrived at a definition of the variable $\hat{x}$ and so it must be in the result set. (2) The variable $\hat{x}$ we are searching for has a function value, and unlike clause (1) there is a non-empty lookup stack. This means the variable on top of the lookup stack, $\hat{x}_{1}$, was a non-local and was pushed on to the non-local stack while searching for the definition of the function it resides in. That function definition, $\hat{x}=\hat{f}$, has now been found, and so we may continue to search for $\hat{x}_{1}$ from the current point in the graph. (3) We have found a definition of $\hat{x}$ but it is defined to be another variable $\hat{x}^{\prime}$. We transitively switch to looking for $\hat{x}^{\prime}$. (4a) We have reached the start of the function body and the variable $\hat{x}$ we are searching for was the formal argument $\hat{x}^{\prime}$. So, continue by searching for $\hat{x}^{\prime}$ from the call site. The IsTop clause constrains this stack frame exit to align with the frame we had last entered (in reverse). (4b) This is the case clause version of the previous. Case clauses can be viewed as inlined functions aligned by program context so use of $\hat{C}$ is not necessary for alignment. (5a) We have reached a return copy which is assigning our variable $x$, so to look for $x$ we need to continue by looking for $x^{\prime}$ inside this function. Push $\hat{c}$ on the stack since we are now entering the body (in reverse) via that call site. For a more accurate analysis, the "provided" line additionally requires that we only "walk back" into function(s) that could have reached this call site; so, we launch a subordinate lookup of $\hat{x}_{f}$ and constrain $\hat{a}_{1}$ accordingly. (6) Here the previous clause is not a match so the search continues at any predecessor node. Note this will chain past function/match call sites which did not return the variable $\hat{x}$ we are looking for. This is sound in a pure functional language; when we address state in Section 5.3, we will enter such a function to verify an alias to our variable was not assigned. (7a) The precondition means we have reached the beginning of a function body and did not find a definition for the variable $\hat{x}$. In this case, we switch to searching for the clause that defined the function body we are exiting, which is $\hat{x}_{f}$, and push $\hat{x}$ on the non-locals stack. Once the defining point of $\hat{x}_{f}$ is found, $\hat{x}$ will be popped from the non-locals stack and we will resume searching for it. The IsTop clause constrains the stack frame being exited to align with the frame we had last entered (in reverse). (7b) This is the case clause variation of the previous; as with (4b) above, the stack is not needed for conditional alignment - there is syntactically only one entry and exit.

\subsection{Abstract Evaluation}

We are now ready to present the single-step abstract evaluation relation on dependency graphs. Like $[28,10]$ etc, it is a graph-based notion of evaluation, but where function bodies are never copied - a single body is shared.

\subsubsection{Active nodes}

In order to preserve standard evaluation order we define the notion of an active node, Active - only nodes with all previous nodes already executed can fire. This serves a purpose similar to an evaluation context in operational semantics [4]. 


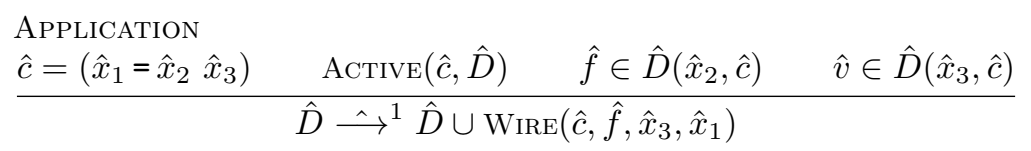

$$
\begin{aligned}
& \text { Record Conditional True } \\
& \frac{\hat{c}=\left(\hat{x}_{1}=\hat{x}_{2} \sim \hat{r} ? \hat{f}_{1}: \hat{f}_{2}\right) \quad \operatorname{Active}(\hat{c}, \hat{D}) \quad \hat{r}^{\prime} \in \hat{D}\left(\hat{x}_{2}, \hat{c}\right) \quad \hat{r} \subseteq \hat{r}^{\prime}}{\hat{D} \hat{\longrightarrow}^{1} \hat{D} \cup \operatorname{WiRe}\left(\hat{c}, \hat{f}_{1}, \hat{x}_{2}, \hat{x}_{1}\right)}
\end{aligned}
$$

$\begin{aligned} & \text { Record Conditional False } \\ & \hat{c}=\left(\hat{x}_{1}=\hat{x}_{2} \sim \hat{r} ? \hat{f}_{1}: \hat{f}_{2}\right) \quad \operatorname{Active}(\hat{c}, \hat{D}) \quad \hat{v} \in \hat{D}\left(\hat{x}_{2}, \hat{c}\right) \quad \hat{v} \text { of form } \hat{r}^{\prime} \text { only if } \hat{r} \nsubseteq \hat{r}^{\prime}\end{aligned}$
$\hat{D} \stackrel{\leftrightarrow}{ }^{1} \hat{D} \cup \operatorname{Wire}\left(\hat{c}, \hat{f}_{2}, \hat{x}_{2}, \hat{x}_{1}\right)$

Figure 11 Abstract Evaluation Rules.

- Definition 3.5. Active $\left(\hat{a}^{\prime}, \hat{D}\right)$ iff path StarT $\ll \hat{a}_{1} \ll \ldots \ll \hat{a}_{n} \ll \hat{a}^{\prime}$ appears in $\hat{D}$ such that no $\hat{a}_{i}$ is of one of the forms $\hat{x}=\hat{x}^{\prime} \hat{x}^{\prime \prime}$ or $\hat{x}=\hat{x}^{\prime} \sim \hat{p} ? \hat{f}: \hat{f}^{\prime}$. We write $\operatorname{Active}\left(\hat{a}^{\prime}\right)$ when $\hat{D}$ is understood from context.

\subsubsection{Wiring}

Recall from Section 2 how function application required the concrete function body to be "wired" directly in to the call site node, and how additional nodes were added to copy in the argument and out the result. The following definition accomplishes this.

- Definition 3.6. Let $\operatorname{WirE}\left(\hat{c}^{\prime}\right.$, fun $\left.\hat{x}_{0} \rightarrow\left(\left[\hat{c}_{1}, \ldots, \hat{c}_{n}\right]\right), \hat{x}_{1}, \hat{x}_{2}\right)=$

$$
\operatorname{Preds}\left(\hat{c}^{\prime}\right) \ll\left(\hat{x}_{0} \stackrel{\hat{c}^{\prime} \downarrow}{=} \hat{x}_{1}\right) \ll \hat{c}_{1} \ll \ldots \ll \hat{c}_{n} \ll\left(\hat{x}_{2} \stackrel{\hat{c}^{\prime} \uparrow}{=} \operatorname{RV}\left(\left[\hat{c}_{n}\right]\right)\right) \ll \operatorname{SucCs}\left(\hat{c}^{\prime}\right) .
$$

$\hat{c}^{\prime}$ here is the call site, and $\hat{c}_{1} \ll \ldots \ll \hat{c}_{n}$ is the wiring of the function body. The Preds/Succs functions (given above in Definition 3.1) reflect how we simply wire to the existing predecessor(s) and successor(s).

Next, we define the abstract small-step relation $\hat{\longrightarrow}^{1}$ on graphs, see Figure 11.

- Definition 3.7. We define the small step relation $\hat{\rightarrow}^{1}$ to hold if a proof exists in the system in Figure 11. We write $\hat{D}_{0} \hat{\longrightarrow}^{*} \hat{D}_{n}$ to denote $\hat{D}_{0} \hat{\longrightarrow}^{1} \hat{D}_{1} \hat{\longrightarrow}^{1} \ldots \hat{\hookrightarrow}^{1} \hat{D}_{n}$.

The evaluation rules are straightforward after the above preliminaries. For application, if $\hat{c}$ is a call site that is an active redex, lookup of the function variable $\hat{x}_{2}$ returns function body $\hat{f}$ and some value $\hat{v}$ can be looked up at the argument position, we may wire in $\hat{f}$ 's body to this call site. Note that $\hat{v}$ is not added to the graph, it is only observed here to constrain evaluation order to be call-by-value. The case clause rules are similar.

\subsection{Decidability}

We begin with the computability of the variable lookup operation, the source of all of the computational complexity of the analysis.

- Lemma 3.8. For any context stack model $\Sigma$ with a finite $\hat{\boldsymbol{C}}$ and computable Push, PoP and IsTop operations, $\hat{D}\left(\hat{x}_{0}, \hat{a}\right)$ is a computable function. 
Proof. This proof proceeds by reduction to the problem of reachability in a push-down system (PDS) accepting by empty stack. A push-down system is a push-down automaton (PDA) with an empty input alphabet; PDA/PDS reachability is polynomial-time [3, 1].

We define a PDS in which each state is a pair between a program point and a context stack (of which there are finitely many); the initial state is the pair $\hat{a} \times \epsilon$. The stack of the PDS corresponds roughly to the lookup stack and the current variable of the lookup operation: each variable of the source program is a member of the stack alphabet and the initial PDS stack is then $\left[\hat{x}_{0}\right]$ for computing $\hat{D}\left(\hat{x}_{0}, \hat{a}\right)$. Each clause in Definition 3.4 (except clause $5 \mathrm{a}$, which is discussed below) directly corresponds to a collection of transitions in the PDS. For instance, suppose $\hat{a}_{1}=\left(\hat{x}^{\prime}=\hat{v}^{\prime}\right)$ and $\hat{a}_{1} \ll \hat{a}_{0}$; then, by clauses 1 and 2 , each node $\hat{a}_{0} \times \hat{C}$ in the PDS transitions to $\hat{a}_{1} \times \hat{C}$ by popping $\hat{x}^{\prime}$ (and pushing no symbols).

Clause 5a of Definition 3.4 requires special handling due to the additional "provided" constraint which must analyze a subordinate invocation of the lookup function. To perform this subordinate lookup in a way that affects the PDS transitions, we include the program's values and the PDS's own states in the PDS stack grammar. A subordinate lookup is started by pushing the current state and the lookup variable onto the stack. When a value is found, it is pushed onto the stack. Finally, every PDS state can transition to any target PDS state by popping the target PDS state from the stack. With some encoded stack reordering, the subordinate lookup returns us to the state in which it started with the resulting value on the top of the stack.

Given the above, it suffices to show that the values given by $\hat{D}\left(\hat{x}_{0}, \hat{a}\right)$ are exactly those of the nodes reachable with an empty stack in the PDS. Let $\hat{V}=\hat{D}\left(\hat{x}_{0}, \hat{a}\right)$ and let $\hat{V}^{\prime}$ be the values in the PDS's reachable nodes. For each $\hat{v} \in \hat{V}, \hat{v} \in \hat{V}^{\prime}$ follows directly by induction on the size of the proof of $\hat{v} \in \hat{V}$, constructing a path through the PDS at each step. For each $\hat{v} \in \hat{V}^{\prime}, \hat{v} \in \hat{V}$ follows directly by induction on the length of any path in the PDS which reaches the node containing $\hat{v}$.

\subsubsection{Some simple context stack models}

A natural family of context stacks is one where stacks are the $k$ latest frames; to also admit the unbounded case we let $k$ range over Nat $\cup \omega$ for $\omega$ the first limit ordinal. We let $\left[\hat{c}^{\prime}, \hat{c}_{1}, \ldots, \hat{c}_{n}\right]\left\lceil k\right.$ denote $\left[\hat{c}^{\prime}, \hat{c}_{1}, \ldots, \hat{c}_{m}\right]$ for $m=\min (k, n)$.

- Definition 3.9. Fixing $k$, we define context stack model $\Sigma_{k}$ as having stack set $\hat{\boldsymbol{C}}$ be the set of all lists of $\hat{c}$ occurring in the program up to length $k$, and with the stack operations as follows:

- $\operatorname{Push}\left(\hat{c}^{\prime},\left[\hat{c}_{1}, \ldots, \hat{c}_{n}\right]\right)=\left[\hat{c}^{\prime}, \hat{c}_{1}, \ldots, \hat{c}_{n}\right]\lceil k$

- $\operatorname{Pop}\left(\left[\hat{c}_{1}, \ldots, \hat{c}_{n}\right]\right)=\left[\hat{c}_{2}, \ldots, \hat{c}_{n}\right]$ if $n>0 ; \operatorname{Pop}([])=[]$.

- $\operatorname{IsTop}\left(\hat{c}^{\prime},\left[\hat{c}_{1}, \ldots, \hat{c}_{n}\right]\right)$ is true if $\hat{c}^{\prime}=\hat{c}_{1}$ or if $n=0$; it is false otherwise.

The term " $k$ DDPA" we use to refer to DDPA with the context stack model $\Sigma_{k}$.

- Lemma 3.10. Fixing $\Sigma$ to some $\Sigma_{k}$ for fixed constant $k, \hat{D}\left(\hat{x}, \hat{a}_{0}\right)$ is computable in polynomial time in the number of nodes in graph $\hat{D}$.

Proof. Let $g$ be the number of nodes in graph $\hat{D}$. By inspection, the PDS built in the proof of Lemma 3.8 will have a number of states which is of order the product of $g$ and the number of context stacks in $\Sigma_{k}$. For $\Sigma_{k}$, the number of stacks is of order $O\left(g^{k}\right)$. Since PDS reachability is computable polynomially in the number of nodes in the PDS, the result immediately follows.

ECOOP 2016 
Note that if $k$ was not fixed and was in fact increasing with the size of the program, it would become exponential.

- Lemma 3.11. Variable lookup is monotonic; that is, for any $\hat{x}$ and $\hat{a}$, if $\hat{D}_{1} \subseteq \hat{D}_{2}$ then $\hat{D}_{1}(\hat{x}, \hat{a}) \subseteq \hat{D}_{2}(\hat{x}, \hat{a})$.

Proof. Variable lookup is encodable as a PDS reachability problem (see Lemma 3.8) and the PDS grows monotonically with the graph $\hat{D}$. PDS reachability grows monotonically with the PDS. Therefore, the set of results from variable lookup grows monotonically with the graph $\hat{D}$.

- Lemma 3.12. The evaluation relation $\hat{\longrightarrow}^{*}$ is confluent.

Proof. By inspection of Figure 11, the single-step rules only add to graph $\hat{D}$. The Active relation is also clearly monotone: any enabled redex is never disabled. Confluence is trivial from these two facts.

- Lemma 3.13. The evaluation relation $\hat{\vec{C}}^{*}$ is terminating, i.e. for any $\hat{D}_{0}$ there exists a $\hat{D}_{n}$ such that $\hat{D}_{0} \hat{\longrightarrow}^{*} \hat{D}_{n}$ and if $\hat{D}_{n} \hat{\longrightarrow}^{*} \hat{D}_{n+1}, \hat{D}_{n}=\hat{D}_{n+1}$. Furthermore, $n$ is polynomial in the size of the initial program.

Proof. By inspection of Figure 11, we have for any step $\hat{D}^{\prime} \hat{\rightarrow}^{1} \hat{D}^{\prime \prime}$ that $\hat{D}^{\prime} \subseteq \hat{D}^{\prime \prime}$. The only new nodes that can be added to $\hat{D}$ in the course of evaluation are the entry/exit nodes $\hat{x}^{\prime} \stackrel{\hat{c} \downarrow}{=} \hat{x} / \hat{x} \stackrel{\hat{c} \uparrow}{=} \hat{x}^{\prime}$, and only one of each of those nodes can exist for each call site (or case clause) / function body pair in the source program: $\hat{c}$ is the call site, and $\hat{x} / \hat{x}^{\prime}$ are variables in that call site and function body source, respectively. So, the number of nodes that can be added is always less then two times the square of the size of the original program. A similar argument holds for added edges.

We let $\hat{D} \downarrow \hat{D}^{\prime}$ abbreviate $\hat{D} \hat{\longrightarrow}^{*} \hat{D}^{\prime}$ such that $\hat{D}^{\prime} \hat{\longrightarrow}^{1} \hat{D}^{\prime}$. We write $e \downarrow \hat{D}$ to abbreviate $\operatorname{EmbeD}(e) \downarrow \hat{D}$; this means the analysis of $e$ returns graph $\hat{D}$. Given the pieces assembled above, it is now easy to prove that the analysis is polynomial-time.

- Theorem 3.14. Fixing $\Sigma$ to be some $\Sigma_{k}$ and fixing some expression e, the analysis result $\hat{D}$, where $e \downarrow \hat{D}$, is computable in time polynomial in the size of $e$.

Proof. By Lemma 3.10, each lookup operation takes poly-time. The evaluation rules are trivial computations besides the required lookups and, by Lemma 3.13, there are polynomially many evaluation steps before termination. Thus $e \downarrow \hat{D}$ is computable in poly-time.

\section{Soundness}

We now establish soundness of the analysis defined in the previous section. In forward program analyses the alignment between the operational semantics and analysis is fairly close and so soundness is not particularly difficult, but here there is a larger gap. We cross this river by throwing a stone in the middle: along with defining a mostly-standard operational semantics we build a graph-based operational semantics which creates a concrete call graph of the program run that is more directly aligned with the analysis. Soundness is then shown by proving the standard and graph-based operational semantics equivalent and by showing the analysis sound with respect to the graph-based operational semantics.

In this section we first present the standard operational semantics, then the graphbased operational semantics and its equivalence to the standard one, and finally we prove soundness. 


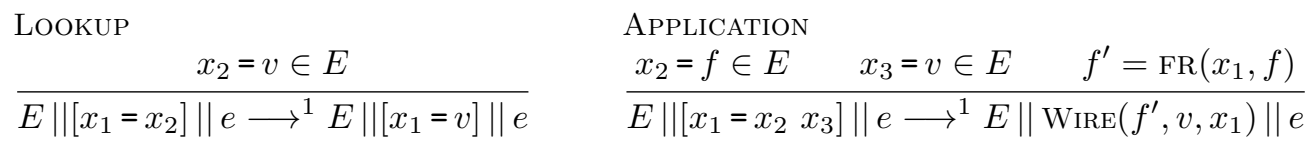

$$
\begin{aligned}
& \text { Record Conditional True } \\
& \qquad \begin{array}{l}
x_{2}=r^{\prime} \in E \quad r \subseteq r^{\prime} \quad f_{1}^{\prime}=\operatorname{FR}\left(x_{1}, f_{1}\right) \\
E\left\|\left[x_{1}=x_{2} \sim r ? f_{1}: f_{2}\right]\right\| e \longrightarrow{ }^{1} E\left\|\operatorname{Wire}\left(f_{1}^{\prime}, r^{\prime}, x_{1}\right)\right\| e
\end{array}
\end{aligned}
$$

Record Conditional False

$\frac{x_{2}=v \in E \quad v \text { of the form } r^{\prime} \text { only if } r \nsubseteq r^{\prime} \quad f_{2}^{\prime}=\operatorname{FR}\left(x_{1}, f_{2}\right)}{E\left\|\left[x_{1}=x_{2} \sim r ? f_{1}: f_{2}\right]\right\| e \longrightarrow^{1} E\left\|\operatorname{WiRE}\left(f_{2}^{\prime}, r^{\prime}, x_{1}\right)\right\| e}$

Figure 12 Small-Step Evaluation.

\subsection{Standard Operational Semantics}

The operational semantics appears in Figure 12, as a small step relation $e \longrightarrow^{1} e^{\prime}$. In many ways the operational semantics is standard, but due to our use of an A-normal form it is neither precisely substitution-based nor environment-based. It is more substitution-based in spirit since function bodies are inlined. Although variable lookup is via an environment, all names in that environment are deterministically freshened and so no variable shadowing ever arises; so, although variable lookup might appear to be dynamically scoped, the absence of shadowing ensures static scoping. This model of evaluation is designed to integrate well with the graph-based semantics of the next sub-section.

We must freshen variables as they are introduced to the expression to preserve the invariant that each variable is uniquely defined and no shadowing occurs. We give a somewhat nonstandard definition of freshening which is deterministic, so as to make alignments easier. We take $\operatorname{FR}\left(x^{\prime}, x\right)$ to yield another variable $x^{\prime \prime}$; we require that $\operatorname{FR}(-,-)$ is injective and that its codomain does not include variables appearing in the initial program. Here, $x$ is the variable to be freshened and $x^{\prime}$ is the point in the program at which it is freshened. For informal illustration, one concrete freshening function could be $\operatorname{FR}\left(x^{\prime}, x\right)=x^{x^{\prime}}$. We overload $\operatorname{FR}\left(x^{\prime}, v\right)$ to indicate the freshening of all variables bound in $v$. The rules for the small step relation are given in Figure 12. Here, wiring is the process of inlining a function body; for this we use the following auxiliary function.

D Definition 4.1. Let $\operatorname{WiRE}\left(\operatorname{fun} x \rightarrow(e), v, x^{\prime}\right)=[x=v]\|e\|\left[x^{\prime}=\operatorname{RV}(e)\right]$.

- Definition 4.2. We define the small step relation $\longrightarrow^{1}$ to hold if a proof exists in the system in Figure 12. We write $e_{0} \longrightarrow^{*} e_{n}$ to denote $e_{0} \longrightarrow^{1} e_{1} \longrightarrow^{1} \ldots \longrightarrow^{1} e_{n}$.

- Definition 4.3. If, for some expression $e$ not of the form $E$, there exists no $e^{\prime}$ such that $e \longrightarrow{ }^{1} e^{\prime}$, then we say that $e$ is stuck. For any $e^{\prime \prime}$ such that $e^{\prime \prime} \longrightarrow{ }^{*} e$, we say that $e^{\prime \prime}$ becomes stuck.

\subsection{Graph-Based Operational Semantics}

We now define the graph-based operational semantics, and prove it to be equivalent to the standard operational semantics just defined. 


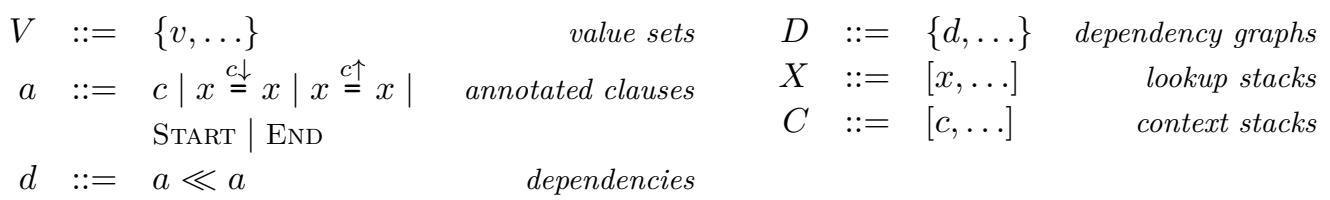

Figure 13 Graph-Based Evaluation Grammar.

The graph-based operational semantics is structurally very similar to the analysis. The primary difference is function bodies here are copied to make new graph structure (as opposed to the analysis, in which only one copy of each function body exists in the graph). Otherwise, the graph-based operational semantics and the analysis are nearly identical, including the use of context stacks, lookup operations, and so on. Since many of these definitions are so similar, we will be much more brief here; we assume that the reader has absorbed the analysis definitions.

The graph-based operational semantics differs from the standard operational semantics of Section 4.1 in the following dimensions:

1. the total ordering of the list becomes a partial ordering here;

2. alias clauses $x=x^{\prime}$ are resolved lazily rather than eagerly, and

3. the graph is monotonically increasing; that is, in each place that the standard semantics replaces a call site, the graph-based semantics builds a path around the call site.

The new grammar constructs needed for the graph-based operational semantics appear in Figure 13.

We write $\operatorname{Embed}(e)$ to denote the initial embedding of an expression $e$ into a dependency graph $D$. This definition is identical to analysis Definition 3.2 with hats removed from the metavariables. Similarly, we also will use the notational sugar of Notation 3.1 in perfect analogy.

\subsubsection{Variable value lookup}

The definition of lookup proceeds with respect to the context stack $C$ which is directly aligned with the corresponding stack $\hat{C}$ of the analysis. This stack is not necessary in the operational semantics (as copying of function bodies removes all of the ambiguity that was in the analysis); we retain it for alignment. Unlike the analysis, we need not bound $C$; we therefore fix the context stack model of the operational semantics to the equivalent of the unbounded model $\Sigma_{\omega}$ in this grammar.

Lookup finds the value of a variable starting from a given graph node; this definition is a near-exact parallel of Definition 3.4 and the reader is referred there for intuitions. We let $D\left(x, a_{0}\right)$ abbreviate $D\left(x, a_{0},[],[]\right)$.

- Definition 4.4. Given graph $D, D\left(x, a_{0}, X, C\right)$ is the function returning the least set of values $V$ satisfying the following conditions:

1. If $a_{1}=(x=v), a_{1} \ll a_{0}$, and $X=[]$, then $v \in V$.

2. If $a_{1}=(x=f), a_{1} \ll a_{0}$, and $X=\left[x_{1}, \ldots, x_{n}\right]$ for $n>0$, then $D\left(x_{1}, a_{1},\left[x_{2}, \ldots, x_{n}\right], C\right) \subseteq$ $V$.

3. If $a_{1}=\left(x=x^{\prime}\right)$ and $a_{1} \ll a_{0}$, then $D\left(x^{\prime}, a_{1}, X, C\right) \subseteq V$.

4a. If $a_{1}=\left(x \stackrel{c \downarrow}{=} x^{\prime}\right), a_{1} \ll a_{0}, c$ is an application clause, and $\operatorname{IsTop}(c, C)$, then $D\left(x^{\prime}, a_{1}, X, \operatorname{Pop}(C)\right) \subseteq V$.

4b. If $a_{1}=\left(x \stackrel{c \downarrow}{=} x^{\prime}\right), a_{1} \ll a_{0}$ and $c$ is a conditional clause, then $D\left(x^{\prime}, a_{1}, X, C\right) \subseteq V$. 


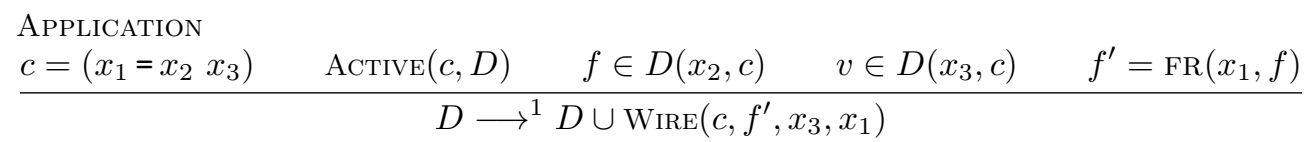

Record Conditional True

$\frac{c=\left(x_{1}=x_{2} \sim r ? f_{1}: f_{2}\right) \quad \operatorname{ACtive~}(c, D) \quad r^{\prime} \in D\left(x_{2}, c\right) \quad r \subseteq r^{\prime} \quad f_{1}^{\prime}=\operatorname{FR}\left(x_{1}, f_{1}\right)}{D \longrightarrow^{1} D \cup \operatorname{WiRe}\left(c, f_{1}^{\prime}, x_{2}, x_{1}\right)}$

Record Conditional False

$c=\left(x_{1}=x_{2} \sim r ? f_{1}: f_{2}\right)$
$\frac{\operatorname{Active}(c, D) \quad v \in D\left(x_{2}, c\right) \quad v \text { of form } r^{\prime} \text { only if } r \nsubseteq r^{\prime} \quad f_{2}^{\prime}=\operatorname{FR}\left(x_{1}, f_{2}\right)}{D \longrightarrow{ }^{1} D \cup \operatorname{WiRe}\left(c, f_{2}^{\prime}, x_{2}, x_{1}\right)}$

Figure 14 Graph Evaluation Rules.

5a. If $a_{1}=\left(x \stackrel{c \uparrow}{=} x^{\prime}\right), a_{1} \ll a_{0}$ and $c=\left(x_{r}=x_{f} x_{v}\right)$, then $D\left(x^{\prime}, a_{1}, X, \operatorname{PusH}(c, C)\right) \subseteq V$, provided fun $x^{\prime \prime} \rightarrow(e) \in D\left(x_{f}, c,[], C\right)$ and $x^{\prime}=\operatorname{RV}(e)$.

5b. If $a_{1}=\left(x \stackrel{c \uparrow}{=} x^{\prime}\right), a_{1} \ll a_{0}$ and $c$ is a conditional clause, then $D\left(x^{\prime}, a_{1}, X, C\right) \subseteq V$.

6. If $a_{1}=\left(x^{\prime \prime}=b\right), a_{1} \ll a_{0}$, and $x^{\prime \prime} \neq x$, then $D\left(x, a_{1}, X, C\right) \subseteq V$.

7a. If $a_{1}=\left(x^{\prime \prime} \stackrel{c \downarrow}{=} x^{\prime}\right), a_{1} \ll a_{0}, x^{\prime \prime} \neq x, c$ is an application clause, and $\operatorname{IsTop}(c, C)$, then $D\left(x_{f}, a_{1}, x \| X, \operatorname{POP}(C)\right) \subseteq V$.

7b. If $a_{1}=\left(x^{\prime \prime} \stackrel{c \downarrow}{=} x^{\prime}\right), a_{1} \ll a_{0}, x^{\prime \prime} \neq x$ and $c$ is a conditional clause, then $D\left(x, a_{1}, X, C\right) \subseteq$ $V$.

where $\operatorname{RV}(e)=x$ if $e=[c, \ldots, x=b]$, i.e. $x$ is the return variable of $e$.

\subsubsection{The evaluation relation}

The definition of an active node, Active, exactly parallels the analysis version of Definition 3.5 , simply remove the hats from the metavariables. We write $\operatorname{Active}\left(a^{\prime}\right)$ when $D$ is understood from context. Wiring is also defined in perfect analogy with Definition 3.6 so is omitted here. The small-step relation $\longrightarrow^{1}$ on graphs is defined in Figure 14. (Note we overload symbol $\longrightarrow^{1}$ for the list and graph operational semantics, it is clear from context which relation is intended.)

- Definition 4.5. We define the small step relation $\longrightarrow^{1}$ to hold if a proof exists in the system in Figure 14. We write $D_{0} \longrightarrow{ }^{*} D_{n}$ to denote $D_{0} \longrightarrow^{1} D_{1} \longrightarrow^{1} \ldots \longrightarrow^{1} D_{n}$.

We also define a notion of "stuckness" for graphs in parallel with the standard operational semantics.

These rules are very similar to the analysis transition rules in Section 3.2; we refer the reader to the descriptions there. Here we comment on a few points unique to the operational semantics not found in the analysis.

We are precise about freshening in these rules. Because the $\operatorname{FR}(x,-)$ function is deterministically freshening based on the call site $x$, it will always generate the same result for the same call site and value. This means that e.g. each use of the Application rule is idempotent and the graph-based operational semantics is deterministic.

Observe how Figure 14 is defining a "reduction" relation which is in fact monotonically increasing, an unusual property compared to standard operational semantics. 


\subsection{Proving Equivalence of Operational Semantics}

The overall proof of soundness relies upon showing that these two systems of operational semantics are equivalent. To demonstrate this, we must show several properties of the graph-based operational semantics which are less obvious than in the standard operational semantics due to the structure of the graph. As stated above, the real differences between these systems are (1) the partial ordering of clauses, (2) lazy resolution of alias clauses, and (3) that the graph grows monotonically instead of replacing applications and conditionals. Nonetheless, the graph representation "runs" in the same way that expressions do: there is a unique clause which is evaluated next, it may cause the introduction of more clauses, and complex clauses (applications and conditionals) are handled by wiring and evaluating the appropriate function body.

We use this reasoning to establish a bisimulation $\cong$ between an expression and its embedding and then show that this bisimulation is preserved as evaluation proceeds. The bisimulation and corresponding preservation proof are tedious and intuitive, so we exclude them for reasons of space. The key equivalence lemma is stated as follows.

- Lemma 4.6 (Equivalence of standard and graph-based semantics). If $e \cong D$, then

1. If $e \longrightarrow^{1} e^{\prime}$ then $D \longrightarrow^{*} D^{\prime}$ such that $e^{\prime} \cong D^{\prime}$.

2. If $D \longrightarrow^{1} D^{\prime}$ then $e \longrightarrow^{*} e^{\prime}$ such that $e^{\prime} \cong D^{\prime}$.

This equivalence is one-to-many to accommodate the differences between the two operational semantics relations. The graph opsems lazily resolves aliases, meaning that several steps of the standard opsems may be required to propagate a value that the graph would find via lookup (Definition 4.4). Likewise, a single lookup step of the standard opsems may require no changes to the graph to maintain bisimulation.

\subsection{Soundness of the Analysis}

We now show that the analysis simulates the graph-based operational semantics, $D \preccurlyeq \hat{D}$. This proof is easy, as the operational semantics graph can be projected on to an analysis graph by inverting the variable freshening function. We formally define this inversion as follows:

- Definition 4.7. The origin of a variable $x$ is $x$ itself if $x$ is not in the codomain of $\operatorname{FR}(-,-)$. Otherwise, let $\operatorname{FR}\left(x^{\prime \prime}, x^{\prime}\right)=x$ (for unique $x^{\prime \prime}$ and $x^{\prime}$, as $\operatorname{FR}(-,-)$ is injective). Then the origin of $x$ is the origin of $x^{\prime}$.

We next define the simulation between evaluation graphs and analysis graphs. The graph operational semantics was designed explicitly to make this simulation direct.

- Definition 4.8 (Simulation relation). Let $f$ be the natural graph-and-clause homomorphism from an evaluation graph $D$ to an analysis graph $\hat{D}$ which maps variables to their origins. We say that $D$ is simulated by $\hat{D}$ (written $D \preccurlyeq \hat{D})$ iff $f(D)=\hat{D}$.

- Lemma 4.9 (Soundness). If $D \preccurlyeq \hat{D}$ and $D \longrightarrow^{1} D^{\prime}$, then $\hat{D} \hat{\curlywedge}^{1} \hat{D}^{\prime}$ with $D^{\prime} \preccurlyeq \hat{D}^{\prime}$.

Proof. By case analysis on the rule used to prove $D \longrightarrow^{1} D^{\prime}$. In particular, the premises of a corresponding rule $\hat{D} \hat{\longrightarrow}^{1} \hat{D}^{\prime}$ can be proven using the premises of $D \longrightarrow D^{\prime}$ and the simulation. 


\section{$5 \quad$ Extensions}

In this section, we outline three extensions: full records, path sensitivity, and mutable state. Our goal here is to show there is no fundamental limitation to the model given in the previous sections: DDPA can in principle be extended to the full feature set of a realistic programming language. Here for simplicity of presentation we take each feature one at a time; in a language where they are all simultaneously added there are additional feature interaction cases that must be addressed.

\subsection{Records}

The "records" presented thus far have no projection operation since there are no values to project; here we outline an extension to records with projection. Consider a lookup of variable $\hat{x}$ : if $\hat{x}$ is defined as $\hat{x}=\hat{x}^{\prime} \cdot \ell$, then this necessitates a record projection; $\hat{x}^{\prime}$ itself may also necessitate further projection, and so on. In the general case, there could be a continuation stack of record projections to be performed. This is similar to the non-local lookup stack of our analysis, and not coincidentally: non-locals may be encoded in terms of records via closure conversion.

In light of this connection, we can extend lookup to support full records using a single stack $\hat{X}$ (now more appropriately thought of as a continuation stack) to handle both data destructors as well as non-local variables. We define the grammar of lookup actions to include variables $\hat{x}$ and projections of the form.$\ell$; we use $\hat{k}$ to range over these lookup actions and extend $\hat{X}$ to all lists of $\hat{k}$. We then augment Definition 3.4 with the following new clauses:

- Definition 5.1. We extend Definition 3.4 to full records by adding the following clauses.

9. If $\hat{a}_{1}=\left(\hat{x}=\left\{\ell_{1}=\hat{x}^{\prime}, \ldots\right\}\right), \hat{a}_{1} \ll \hat{a}_{0}, \hat{X}=\left[\cdot \ell_{1}, \hat{k}_{2}, \ldots, \hat{k}_{n}\right]$, then $\hat{D}\left(\hat{x}^{\prime}, \hat{a}_{1},\left[\hat{k}_{2}, \ldots, \hat{k}_{n}\right], \hat{C}\right) \subseteq \hat{V}$.

10. If $\hat{a}_{1}=\left(\hat{x}=\hat{x}^{\prime} \cdot \ell\right), \hat{a}_{1} \ll \hat{a}_{0}$, and $\hat{X}=\left[\hat{k}_{1}, \ldots, \hat{k}_{n}\right]$, then $\hat{D}\left(\hat{x}^{\prime}, \hat{a}_{1},\left[\cdot \ell, \hat{k}_{1}, \ldots, \hat{k} n\right], \hat{C}\right) \subseteq \hat{V}$.

We also extend each of the clauses of Definition 3.4 to address lookup actions generally (rather than requiring lookup stacks to consist only of variables). Clause 10 above introduces the projection action.$\ell$ when we discover that we will need to project from the variable we find; clause 9 eliminates this projection action when the corresponding record value is found. Note that record pattern matching would also be desirable syntax, and would be possible by extending clause 8 of the original lookup definition; for brevity, we skip that here.

\subsection{Filtering for path sensitivity}

An additional level of expressiveness we desire from records is the ability to filter out paths that cannot have been taken. Consider, for instance, the recursive example in Figure 8. This example appears to be unsafe based on the analysis result: on line 7 , we project 1 from the function's argument. The condition on line 4 makes this safe - only records with 1 labels may reach line 7 - but the analysis is unaware of this and so erroneously believes that the record \{\} may reach that point.

We address this incompleteness using filters: sets of patterns which the value must match in order to be considered relevant. In the above example, for instance, looking up $\mathrm{v}$ from line 7 leads us to look up a from line 4. Because our path moved backwards through the first clause of a conditional, however, we know that we may ignore any values we discover 
which do not match the $\{1\}$ pattern. This is key to enabling path sensitivity and preventing spurious errors; with filters, DDPA correctly identifies the recursion example as safe.

We can formalize path sensitivity in DDPA by keeping track of sets of accumulated patterns in our lookup function, and disallowing matches not respecting the patterns they passed through. We use $\Pi^{+}$and $\Pi^{-}$to range over sets of patterns which a discovered value must or must not match, respectively. Formally, path-sensitive DDPA is possible by modifying the lookup function as follows:

- Definition 5.2. We modify Definition 3.4 by adding to the lookup function two parameters of the forms $\Pi^{+}$and $\Pi^{-}$. Each existing clause of that definition passes these arguments unchanged. We additionally replace clauses 1 and $4 \mathrm{~b}$ of Definition 3.4 with:

1. If $\hat{a}_{1}=(\hat{x}=\hat{v}), \hat{a}_{1} \ll \hat{a}_{0}, \hat{X}=[]$, and $\hat{v}$ matches all patterns in $\Pi^{+}$and no patterns in $\Pi^{-}$, then $\hat{v} \in \hat{V}$.

4b1. If $\hat{a}_{1}=\left(\hat{x}^{\prime} \stackrel{\hat{c} \downarrow}{=} \hat{x}_{1}\right), \hat{a}_{1} \ll \hat{a}_{0}, \hat{c}=\left(\hat{x}_{2}=\hat{x}_{1} \sim \hat{p} ? \hat{f}_{1}: \hat{f}_{2}\right), \hat{f}_{1}=$ fun $\hat{x}^{\prime}->(\hat{e})$, and $\hat{x} \in\left\{\hat{x}_{1}, \hat{x}^{\prime}\right\}$, then $\hat{D}\left(\hat{x}^{\prime}, \hat{a}_{1}, \hat{X}, \hat{C}, \Pi^{+} \cup\{\hat{p}\}, \Pi^{-}\right) \subseteq \hat{V}$.

4b2. If $\hat{a}_{1}=\left(\hat{x}^{\prime} \stackrel{\hat{c} \downarrow}{=} \hat{x}_{1}\right), \hat{a}_{1} \ll \hat{a}_{0}, \hat{c}=\left(\hat{x}_{2}=\hat{x}_{1} \sim \hat{p}\right.$ ? $\left.\hat{f}_{1}: \hat{f}_{2}\right), \hat{f}_{2}=$ fun $\hat{x}^{\prime}->(\hat{e})$, and $\hat{x} \in\left\{\hat{x}_{1}, \hat{x}^{\prime}\right\}$, then $\hat{D}\left(\hat{x}^{\prime}, \hat{a}_{1}, \hat{X}, \hat{C}, \Pi^{+}, \Pi^{-} \cup\{\hat{p}\}\right) \subseteq \hat{V}$.

4b3. If $\hat{a}_{1}=\left(\hat{x}^{\prime} \stackrel{\hat{c} \downarrow}{=} \hat{x}_{1}\right), \hat{a}_{1} \ll \hat{a}_{0}, \hat{c}=\left(\hat{x}_{2}=\hat{x}_{1} \sim \hat{p}\right.$ ? $\left.\hat{f}_{1}: \hat{f}_{2}\right)$, and $\hat{x} \notin\left\{\hat{x}_{1}, \hat{x}^{\prime}\right\}$, then $\hat{D}\left(\hat{x}^{\prime}, \hat{a}_{1}, \hat{X}, \hat{C}, \Pi^{+}, \Pi^{-}\right) \subseteq \hat{V}$.

We then redefine $\hat{D}(\hat{x}, \hat{a})$ to mean $\hat{D}(\hat{x}, \hat{a},[],[], \emptyset, \emptyset)$.

Revised clause 1 shows how the filters are used: any value not matching the positive filter is filtered out, and oppositely for the negative filter. The original clause 4 was the case where we reached the start of a function and search variable $\hat{x}$ was passed as the parameter; in that case, the clause continued by searching for the argument at the call site. The original clause $4 \mathrm{~b}$ was the case where we reached the start of a case clause and search variable $\hat{x}$ was passed as the parameter; in that case, the clause continued by searching for the argument at the call site. Here, we have separated that rule into two cases. In $4 \mathrm{~b} 1$, the function was the first branch of a conditional, so we know that any discovered value is only relevant if it matches the conditional's pattern. Thus, we add the pattern to the filter set to constrain it so. Clause $4 \mathrm{~b} 2$ is the opposite case.

\subsection{State}

Lookup in the presence of state may also be performed using only a call graph. We consider here a variation of the presentation language which includes OCaml-style references with ref $x / ! x / x<-x$ syntax. There are several subtle issues that must be addressed. First, a simple linear search may not always give the correct answer; a cell containing a cell could have the inner cell mutated after the outer cell, which is out of order compared to the control flow sequence. So, we must define a branching search for references. Second, aliases may arise in the form of different variable names referring to the same cell. Lookup must not overlook aliases; otherwise, the lookup will be inaccurate and incomplete. So, explicit alias testing is needed to verify proper values are not being passed by.

Not only may DDPA be adapted to address state, but the alias analysis itself is not difficult using the same lookup routine. The following definition revises the lookup operation for state.

- Definition 5.3. Definition 3.4 is extended to a stateful language by adding the following clauses. 
9. If $\hat{a}_{1}=\left(\hat{x}=! \hat{x}^{\prime}\right), \hat{a}_{1} \ll \hat{a}_{0}$, then letting $\hat{V}^{\prime}=\hat{D}\left(\hat{x}^{\prime}, \hat{a}_{1},[], \hat{C}\right)$, for each ref $\hat{x}^{\prime \prime} \in \hat{V}^{\prime}$, $\hat{D}\left(\hat{x}^{\prime \prime}, \hat{a}_{1}, \hat{X}, \hat{C}\right) \subseteq \hat{V}$.

10a. If $\hat{a}_{1}=\left(\hat{x}_{1}^{\prime}=\hat{x}_{2}^{\prime}<-\hat{x}_{3}^{\prime}\right), \hat{a}_{1} \ll \hat{a}_{0}$, and MayAlias $\left(\hat{x}, \hat{x}_{2}^{\prime}, \hat{a}_{1}, \hat{C}\right)$, then $\operatorname{ref} \hat{x}_{3}^{\prime} \in \hat{V}$.

10b. If $\hat{a}_{1}=\left(\hat{x}_{1}^{\prime}=\hat{x}_{2}^{\prime}<-\hat{x}_{3}^{\prime}\right), \hat{a}_{1} \ll \hat{a}_{0}$, and MayNotAlias $\left(\hat{x}, \hat{x}_{2}^{\prime}, \hat{a}_{1}, \hat{C}\right)$, then $\hat{D}\left(\hat{x}, \hat{a}_{1}, \hat{X}, \hat{C}\right) \subseteq$ $\hat{V}$.

In these clauses, the terms MayAlias and MAYNotAlias refer to the following predicates:

- $\operatorname{May} \operatorname{Alias}\left(\hat{x}_{1}, \hat{x}_{2}, \hat{a}, \hat{C}\right)$ holds iff $\hat{V}^{\prime}=\hat{D}\left(\hat{x}_{1}, \hat{a},[], \hat{C}\right), \hat{V}^{\prime \prime}=\hat{D}\left(\hat{x}_{2}, \hat{a},[], \hat{C}\right)$, and $\exists \operatorname{ref} \hat{x}^{\prime \prime} \in$ $\left(\hat{V}^{\prime} \cap \hat{V}^{\prime \prime}\right)$

- $\operatorname{MayNotAlias}\left(\hat{x}_{1}, \hat{x}_{2}, \hat{a}, \hat{C}\right)$ holds iff $\hat{V}^{\prime}=\hat{D}\left(\hat{x}_{1}, \hat{a},[], \hat{C}\right), \hat{V}^{\prime \prime}=\hat{D}\left(\hat{x}_{2}, \hat{a},[], \hat{C}\right)$, and $\hat{V}^{\prime} \neq$ $\hat{V}^{\prime \prime}$ or $\hat{V}^{\prime} \neq\left\{\right.$ ref $\left.\hat{x}^{\prime}\right\}$ or $\hat{V}^{\prime \prime} \neq\left\{\right.$ ref $\left.\hat{x}^{\prime \prime}\right\}$

Clause 9 handles dereferencing. It finds the ref values which may be in $\hat{x}^{\prime}$ at this point in the program; it then returns to this point to find all of the values that those variables may contain. This new task is necessary since we want the value at the point the ! happened.

Clauses 10a and 10b address cell updates. Clause 10a determines if the updated cell in $\hat{x}_{2}^{\prime}$ may alias the cell we are looking up; if this is the case, the value assigned by the cell update may be our answer. Clause $10 \mathrm{~b}$ addresses the case in which the updated cell may be different from the target of our lookup. This happens when the lookups of each variable yield different results or when they result in multiple cells - even if the sets of cells are equal, the orders in which the program modifies the cells might differ, so we take the conservative approach and call them different. MAyAlias and MayNotAlias can be simultaneously satisfiable; when that happens, the analysis explores both clauses 10a and $10 \mathrm{~b}$.

Along with the above modifications, the existing clauses 5 and 6 need to be extended to support state. As written, clause 6 allows us to skip by call sites and pattern matches whose output do not match the variable for which we are searching. This is sound in a pure system, but in the presence of side-effects we must explore these clauses to ensure that they did not affect the cell we are attempting to dereference. We thus modify clause 6 by prohibiting $\hat{b}$ from being a call site or pattern match. We require a new clause similar to clause 5 but for the case in which the search variable does not match the output variable. In that case, we proceed into the body of the function but in a "side-effect only" mode: we skip by every clause which is not a cell assignment or does not lead to one. We leave side-effect only mode once we leave the beginning of the function which initiated it.

\section{Implementation}

We have developed a proof-of-concept implementation of DDPA [17]; a copy of the implementation is archived as an artifact with this paper. This implementation analyzes the presentation language given in Section 2 extended to the proper record semantics described in Section 5. The implementation correctly generates all control-flow graphs and value lookup results given in the overview.

Although the proof-of-concept implementation demonstrates the behavior of DDPA, it is far from efficient and we are presently developing a performant implementation. Our implementation follows the lookup algorithm outlined in the proof of Lemma 3.8: we construct a push-down automaton modeling a non-deterministic backwards walk of the DDPA graph and then analyze this PDA for states reachable with an empty stack. There is a wealth of literature on PDA reachability algorithms [3, 1] which we can utilize to gain efficiency; in particular, [3] includes an algorithm for eliminating spurious nodes and edges which we have already integrated into the proof-of-concept implementation due to its simplicity.

ECOOP 2016 


\section{Related Work}

\subsection{CFL-reachability}

The core idea of our analysis can be viewed as extending first-order demand-driven CFLreachability analyses [6] to the higher-order case: the analysis is centered around using a CFG, calls and returns are aligned, and lookup is computed lazily. Two issues make the higher-order analysis challenging: the CFG needs to be computed on-the-fly due to the presence of higher-order functions, and non-local variable lookup is subtle. The aforecited demand-driven analyses delve further into the trade-off between active propagation and demand-driven lookup, and this is something we plan to explore in future work. There are many other first-order program analyses with a demand-driven component; several use Datalog-style specification formats [19] including [29, 23].

Higher-order program analyses have been built which also incorporate call-return alignment $[26,8]$, but these higher-order analyses are not demand-driven and have a different algorithmic basis. We now contrast DDPA with these and other higher-order program analyses.

\subsection{Higher-order program analysis}

As mentioned in the Introduction, higher-order program analyses today are generally constructed by abstract interpretation [2], a finitization process on the operational semantics. We refer to these analyses as forward analyses to contrast with the backward-looking, demand-driven approach of DDPA.

In the most basic forward analysis, a new state is created for each new program point/store/environment, and the number of states grows too rapidly to be practical. Modifications including store widening, abstract garbage collection, polyvariance, and call-return alignment are thus added as means to obtain a better trade-off of expressiveness vs size of state space. DDPA in some sense comes from the opposite direction: the global state information is a graph isomorphic to the CFG which is polynomial in size and will be smaller than the state space of any forward analysis. However, variable lookup is of high complexity and we need to "spend" more space to make it more efficient.

We will expand by considering the different dimensions of analysis expressiveness in turn. We begin with flow-sensitivity, meaning the analysis is sensitive to the order of assignment operations. Forward analyses are initially flow-sensitive since each abstract state has its own store, but the standard store widening optimization unifies these stores and eliminates flowsensitivity. One alternative is abstract garbage collection $[14,8]$, which requires a per-node store (since, if the store were global, there would be no garbage). Abstract garbage preserves flow-sensitivity while reducing abstract states caused by local data, but state explosions still occur with persistent data. DDPA is flow-sensitive and garbage-free by construction: it is flow-sensitive because variables are looked up with respect to a program point, and it is garbage-free vacuously (as no stores are ever created). In a practical implementation of DDPA, the caching of values will bring it closer to an abstract garbage collection approach, but abstract garbage collection is bottom-up as opposed to top-down: rather than deleting items proved unneeded, items are added only if they may be needed in the future.

Another important dimension of expressiveness is polyvariance: whether functions can take on different forms in different contexts of use. The classic higher-order polyvariance model is $k$ CFA [22], which copies contours in analogy to forall-elimination in a polymorphic type system. But there are many routes to behavior that appears as polyvariance. Without store widening, flow-sensitivity alone can distinguish calling context and provide some 
polyvariance. Additionally, call-return alignment can provide different contexts for different function calls. The example we gave in Figure 3, for instance, needs only call-return alignment to give polyvariant behavior in DDPA.

Call-return alignment was first explored in a higher-order context in subtype constraint theories [18]; this work also uses the demand-driven nature of CFL-reachability to optimize lookup. However, it is flow-insensitive, uses let-polymorphism only, and does not align nonlocal variables, and so is not in the same category of analysis. In the context of abstract interpretation, the first such algorithm was CFA2 [26], which was subsequently extended and refined in $k$ PDCFA [8]. We compare primarily with the more recent $k$ PDCFA here.

An important observation of [8] regarding $k$ PDCFA was that, in the presence of abstract garbage collection, it is not possible to have an arbitrary stack for aligning calls and returns. That paper proposes a regular expression modeling of call stacks to make the analysis computable. We have a similar problem: our analysis finitizes one of its two stacks to conform to a PDA encoding. We define the family of analyses $k$ DDPA (Definition 3.3) where $k$ is the maximum stack depth for call-return alignments. Our finitization here is simpler than the regular expression approach of $k$ PDCFA; the regular expression approach may work in our context and is a topic for future investigation.

DDPA achieves greater polyvariance solely from call-return alignment when compared with $k$ PDCFA or CFA2. In $k$ PDCFA, stack alignment can be used to provide a different context for function parameters, but non-local variables get no such advantage since they are not in the local stack context. In DDPA, the stack context is still applicable to nonlocal variable lookup as shown in Figure 5. In fact, we conjecture that, for a program with a maximal lexical nesting depth of $c$, the analysis $(k+c)$ DDPA will be at least as expressive as $k$ CFA (and should be more expressive due to alignment of calls and returns). The additional $c$ levels are needed because, in the worst case, each extra lexical level requires the lookup of a non-local variable and thus a position on the lookup stack. This way, DDPA achieves with only stack alignment what forward analyses need both stack alignment and polyvariant contours to accomplish.

The run-time complexity of $k$ DDPA can also be framed in terms of the expressiveness of non-local variable polyvariance. It is shown in [15] how non-locals are the (only) source of exponential behavior in $k$ CFA [24]; in particular, if lexical nesting were assumed to be of some constant depth not tied to the size of the program, $k$ CFA would not be exponential. The complexity of $k$ DDPA comes from the other direction: for any fixed $k$, the algorithm $k$ DDPA is polynomial; but $k$ needs to be increased by one for each level of stack alignment we wish to achieve in non-local lookup. For pathologically nested programs, $k$ must be on the order of the size of the program for $k$ DDPA to avoid imprecision due to non-local lookup. Because $k$ DDPA's complexity is exponential in $k$, such a non-local-precise $k$ DDPA would be exponentially complex in that pathological case.

Related to this are provably polynomial context-sensitive analyses which, like $k$ DDPA, restrict context-sensitivity in the case of high degree of lexical nesting [7, 15]. $m$ CFA [15] is a polyvariant analysis hierarchy for functional languages that is provably polynomial in complexity. This is achieved by an analysis that "in spirit" is working over closure-converted source programs: by factoring out all non-local variable references, the worst-case behavior has also been removed. Unfortunately, this also affects the precision of the analysis: nonlocals that are distinguished in $k \mathrm{CFA}$ are merged in $m \mathrm{CFA}$. In $k \mathrm{DDPA}$, the level of non-local precision is built into the constant $k$ of how deep the run-time stack approximation is, so more precision is achieved as $k$ increases. $m$ CFA does not have this property: non-locals will always be monomorphised for any $m$. 
Our current implementation is a proof-of-concept only; we plan to investigate ideas in $[11,8,9]$ and other papers for more performant PDA reachability algorithms. Overall the trade-offs in performance and expressiveness are subtle and implementations will be necessary to decide how practically useful DDPA is.

\section{Conclusions}

In this paper, we have developed a demand-driven program analysis (DDPA) for higher-order programs which is centered around production of a call graph. DDPA needs only the call graph to look up variable values; the specification does not maintain any other structures. DDPA can be viewed as the adaptation of previous CFL-reachability-based demand-driven program analyses to higher-order programs. This adaptation required two key changes. First, it was necessary to incrementally construct the control-flow graph as the analysis proceeded; second, the lookup of non-local variables required special handling.

We believe DDPA shows promise primarily because it represents a significantly different approach compared with other higher-order analyses. A high-level analogy can be made with eager and lazy programming languages: it is a fundamental decision in language design which approach to take and there are significant trade-offs. We believe the demand-driven side of higher-order program analyses deserves further exploration.

We have established a polynomial-time bound on a higher-order program analysis which is both flow- and context-sensitive. The reduced global state size holds out promise for program verification tools: the fewer the states, the less overwhelming the workload will be for a model checker, theorem prover, or other verification strategy.

We present preliminary results here from a simple implementation to serve as a confirmation of correctness, but our implementation needs tuning and would benefit from a headto-head comparison with some state-of-the-art analyses. Although DDPA's value lookup is novel, it shares the task of PDA reachability with the existing program analysis literature and so a rich body of work is available to be utilized in implementing DDPA efficiently.

We believe the analysis should scale well to other language features, and have presented outlines for extensions of the basic analysis to deep data structures, path-sensitivity, and state; we leave efficient decision procedures for the latter two to future work. We also intend to explore the development of extensions for other language features: exceptions and other control operators, concurrency, and modularity to name a few.

Acknowledgements. We thank Alex Rozenshteyn for motivating our interest in AAM [25] and related papers which then led to our formulation of DDPA. We thank David Van Horn for discussions helping us better understand the AAM work. We also thank Leandro Facchinetti for his detailed review of this paper and his contributions to the accompanying artifact.

\section{References}

1 Ahmed Bouajjani, Javier Esparza, and Oded Maler. Reachability analysis of pushdown automata: Application to model-checking. In CONCUR'97, 1997.

2 Patrick Cousot and Radhia Cousot. Abstract interpretation: A unified lattice model for static analysis of programs by construction or approximation of fixpoints. In POPL, 1977.

3 Christopher Earl, Matthew Might, and David Van Horn. Pushdown control-flow analysis of higher-order programs. In Workshop on Scheme and Functional Programming, 2010.

4 M. Felleisen and R. Hieb. The revised report on the syntactic theories of sequential control and state. Theoretical Computer Science, 1992. 
5 Cormac Flanagan, Amr Sabry, Bruce F. Duba, and Matthias Felleisen. The essence of compiling with continuations. In PLDI, 1993.

6 Susan Horwitz, Thomas Reps, and Mooly Sagiv. Demand interprocedural dataflow analysis. In Foundations of Software Engineering, 1995.

7 Suresh Jagannathan and Stephen Weeks. A unified treatment of flow analysis in higherorder languages. In POPL '95, 1995.

8 J. Ian Johnson, Ilya Sergey, Christopher Earl, Matthew Might, and David Van Horn. Pushdown flow analysis with abstract garbage collection. JFP, 2014.

9 John Kodumal and Alex Aiken. The set constraint/CFL reachability connection in practice. In PLDI, 2004.

10 John Lamping. An algorithm for optimal lambda calculus reduction. In POPL, 1990.

11 Yi Lu, Lei Shang, Xinwei Xie, and Jingling Xue. An incremental points-to analysis with CFL-reachability. In Compiler Construction, 2013.

12 Jan Midtgaard. Control-flow analysis of functional programs. ACM Comput. Surv., 2012.

13 Matthew Might. Abstract interpreters for free. In Proceedings of the 17th International Conference on Static Analysis, 2010.

14 Matthew Might and Olin Shivers. Improving flow analyses via ГCFA: Abstract garbage collection and counting. In ICFP, Portland, Oregon, 2006.

15 Matthew Might, Yannis Smaragdakis, and David Van Horn. Resolving and exploiting the $k$-CFA paradox: Illuminating functional vs. object-oriented program analysis. In PLDI, 2010.

16 Flemming Nielson, Hanne R. Nielson, and Chris Hankin. Principles of Program Analysis. Springer-Verlag New York, Inc., Secaucus, NJ, USA, 1999.

17 Zachary Palmer and Leandro Facchinetti. Odefa proof-of-concept implementation. https: //github.com/JHU-PL-Lab/odefa-proof-of-concept, 2015. Accessed: 2015-10-11.

18 Jakob Rehof and Manuel Fähndrich. Type-base flow analysis: from polymorphic subtyping to CFL-reachability. In POPL, New York, NY, USA, 2001.

19 Thomas Reps. Demand interprocedural program analysis using logic databases. In Application of Logic Databases, 1994.

20 Thomas Reps. Shape analysis as a generalized path problem. In PEPM, 1995.

21 Thomas Reps, Susan Horwitz, and Mooly Sagiv. Precise interprocedural dataflow analysis via graph reachability. In $P O P L, 1995$.

22 Olin Shivers. Control-Flow Analysis of Higher-Order Languages. PhD thesis, CarnegieMellon University, 1991. TR CMU-CS-91-145.

23 Yannis Smaragdakis and Martin Bravenboer. Using Datalog for fast and easy program analysis. In Datalog Reloaded: First International Workshop, 2011.

24 David Van Horn and Harry G. Mairson. Deciding $k$ CFA is complete for EXPTIME. In ICFP, 2008.

25 David Van Horn and Matthew Might. Abstracting abstract machines. In ICFP, 2010.

26 Dimitrios Vardoulakis and Olin Shivers. CFA2: A context-free approach to control-flow analysis. In European Symposium on Programming, 2010.

27 Dimitrios Vardoulakis and Olin Shivers. Pushdown flow analysis of first-class control. In ICFP, 2011.

28 Christopher P. Wadsworth. Semantics and Pragmatics of the Lambda-calculus. PhD thesis, University of Oxford, 1971.

29 Xin Zhang, Mayur Naik, and Hongseok Yang. Finding optimum abstractions in parametric dataflow analysis. In PLDI, 2013. 